\title{
Analysis of selected parking characteristics in the Paid Parking Zone - a Case Study Based on City in Poland
}

\author{
Agata Kurek $^{1}$, Elżbieta Macioszek ${ }^{1}$ \\ ${ }^{1}$ Silesian University of Technology, Faculty of Transport and Aviation Engineering \\ 40-019 Katowice, Krasińskiego 8 Street, Poland \\ Agata.Kurek@polsl.pl; Elzbieta.Macioszek@polsl.pl
}

\begin{abstract}
Paid Parking Zones (PPZ) currently operate in medium and large cities. Such zones are treated as a tool influencing changes in the transport behavior of residents, prompting them to change a car to alternative means of transport. The basic condition for the efficient management of PPZ is the availability of information on parking characteristics in the zone i.a. parking time, rotation indicator, use of parking space, etc. This paper presents the results of the analysis of selected parking characteristics in the PPZ in Gliwice. These characteristics can be used to assess the functioning of the PPZ, but also to improve the effectiveness of the tools used to manage the PPZ zone. The analysis was performed as a part of research work entitled "Analysis of parking characteristics in the conditions of SPP and DIP functioning in selected areas of GZM cities".
\end{abstract}

Keywords: Paid Parking Zone, parking, Dynamic Parking Information, traffic engineering, road transport.

\section{Introduction}

The last stage of the journey by car is the search for an empty parking space. This process may increase congestion in the area near parking as well as adversely affects the level of road safety and the natural environment [1-4]. The introduction of Paid Parking Zones (PPZ) may effectively discourage drivers from traveling by car to the city center [5], increase the turnover of parking spaces, which in turn allows for better use of parking spaces and increases their availability [6]. Moreover, the search for an empty parking space forces drivers to divide their attention, because the driver must simultaneously focus on driving the vehicle by the road traffic regulations and at the same time must analyze the dynamically changing condition of the environment to find a parking space [7,8]. A solution often used in this case is the introduction of the Dynamic Parking Information (DIP) system, which guides the driver to an empty parking space $[9,10]$. Dynamic parking information provides drivers moving around the city with messages about the level of occupancy of parking spaces using electronic tables located on streets leading car traffic to the parking zone. Dynamic transmit this information to drivers commuting to the zone is associated with the need to continuously receive data on the current use of parking spaces on streets of the paid parking zone. Parking information systems are most often elements of Intelligent Transport Systems (ITS).

The number of parking spaces in the SPP should be adjusted to the demand for parking in a given area in specific periods of the day, days of the week, and months of the year. Due to the uneven distribution of traffic during the day, week, month, and year, parking may be overcrowded or have little or no parked vehicles [11]. The results of the research presented in the literature [12] indicate that most people use parking when traveling to and from work. This paper presents the results of the analysis of selected parking characteristics in the SPP in 2019 in Gliwice.

\section{Parking Measures}

The basic measures of parking are [11]:

- Parking absorbency - understood as the largest number of parking vehicles during the measurement period in the area of analyzes. It can be given by the street,

- parking accumulation - it is a measure of parking intensity, defined as the number of vehicles parking simultaneously in the analyzed area,

- peak parking fill rate - the ratio of peak accumulation to parking capacity,

- estimated reserves of parking spaces - most often determined based on an area inventory, relating to public space (not including private parking), 
- share of vehicles parked illegally,

- rotation indicator - it is the degree of use of the same space by vehicles in the analyzed measurement period.

The method of obtaining parking data varies and depends on the purposes for which the obtained data will be used. Some data can be collected using automated data collection technologies, but some still require a traditional research approach.

\section{Research Area}

The analysis of selected parking characteristics in the Paid Parking Zone was performed for Gliwice (Poland) in 2019. The data for analysis was obtained from the Traffic Control Center (in polish: CSR) in Gliwice, which monitors and supervises road traffic using ITS systems. The analysis used data from $18 \mathrm{PPZ}$ segments equipped with parking sensors and DIP tables (Fig. 1). When a vehicle is detected in a parking space by a magnetic sensor, information is transmitted to concentrators by disturbing the magnetic field generated by the induction loop. Then, these data are sent to the controller via a fiber-optic network, and information about the number of empty parking spaces is displayed on DIP tables. Information on the occupancy of parking spaces is also available to users of the ITS Gliwice application. Among the drivers who use the zone, some people appear there sporadically, e.g. in connection with: dealing with official matters, shopping, tourism, etc., people regularly coming to the city center from other districts and towns (obligatory trips, most often caused by professional or educational needs) and people living in the zone. The drivers of the latter group function the opposite of the drivers of the remaining user groups, i.e. they usually stay in the zone to leave it when the vehicle user leaves for a specific purpose. Different purposes of travel and the way vehicles are used, especially concerning parking, cause city authorities to introduce various forms of parking fees for selected groups of users (i.e. one-off payments, subscriptions).

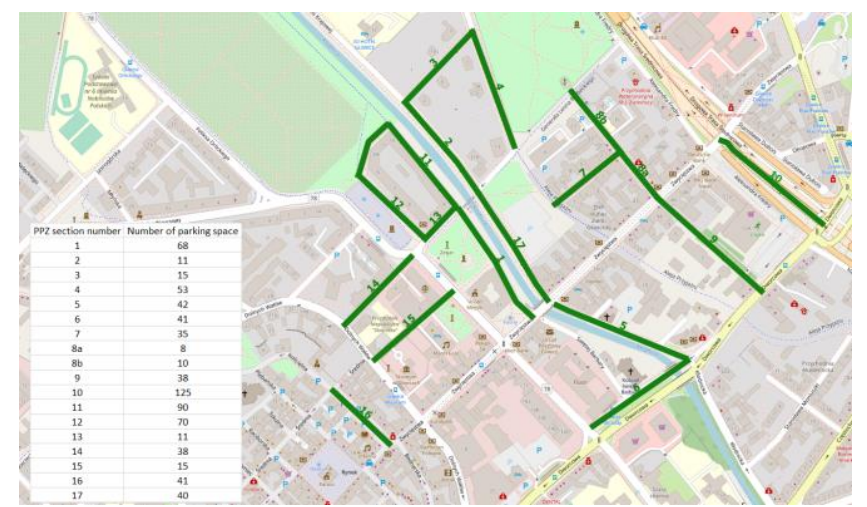

Fig. 1. The Paid Parking Zone with the division of zones into segments with the number of parking spaces

\section{Analysis of the use of parking spaces in time in the Paid Parking Zone in Gliwice}

Parking measurements are one of the important elements helpful in planning the servicing of a given area. The use of in time parking spaces in each segment of the Paid Parking Zone included the analysis of such characteristics as:

- the average number of vehicles in all months of the year on particular days of the week (Fig. 2), in all hours of the day on particular days of the week in the most loaded month (i.e. December) (Fig. 4), in all hours on each day in December (Fig. 6),

- rotation indicator for parking spaces in all months of the year on particular days of the week (Fig. 3), in all hours of the day on particular days of the week in the most loaded month (i.e. December) (Fig. 5), in all hours on each day of December (Fig. 7).

Figures 2-7 present the selected results of the analyzes. The greatest use of the parking space and the rotation indicator of the analyzed segments took place in December taking into account all days of the week (Fig. 6-7). While the least use of the parking space and the rotation indicator of the analyzed segments took place in April. Figure 4 show that the greatest use of analyzed parking spaces occurred on working days, from 07:00 to 16:00. The highest rotation 
indicator in analyzed parking spaces occurred on working days, from 09:00 to 18:00. The fewest vehicles were parked in the morning and night hours. Short parking time for drivers indicates that the drivers who use the analyzed parking are people whose purpose is e.g. official matters, shopping, family social meetings or dealing with short term issues in the city center. On 16-20.12.2020 (Monday-Friday), the use of parking space during the day was characterized by greater diversification than on the other days of the month. This fact may be related to the occurrence of Christmas the following week.

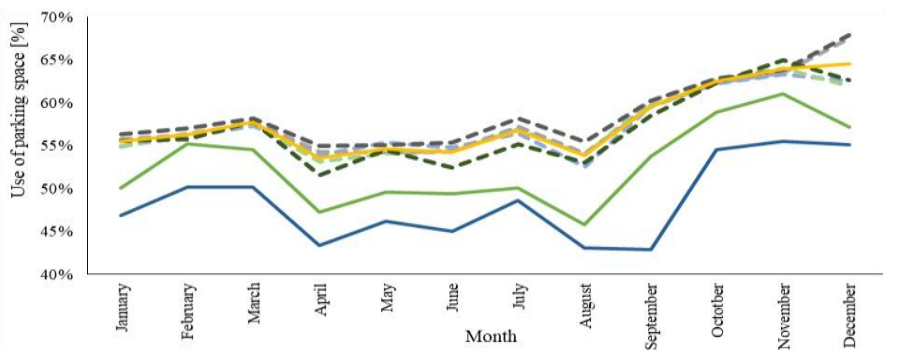

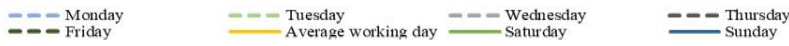

Fig. 2. Average use of parking space in all sections in every month on a particular day of the week

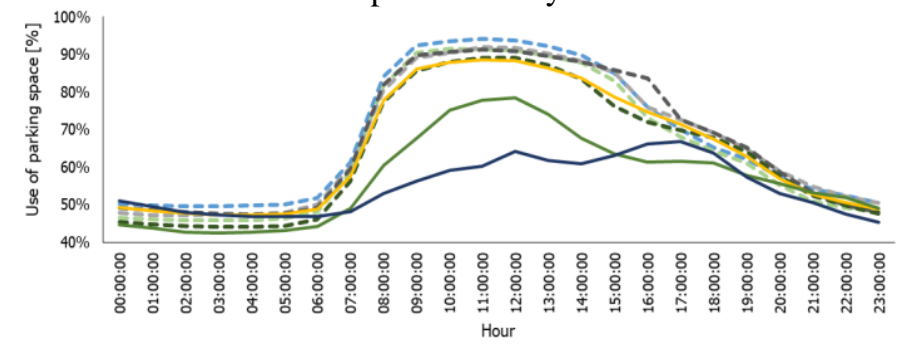

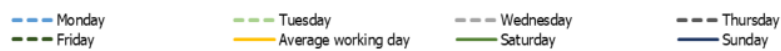

Fig. 4. Average use of parking space in all sections in December at every hour of the day and on a particular day of the week

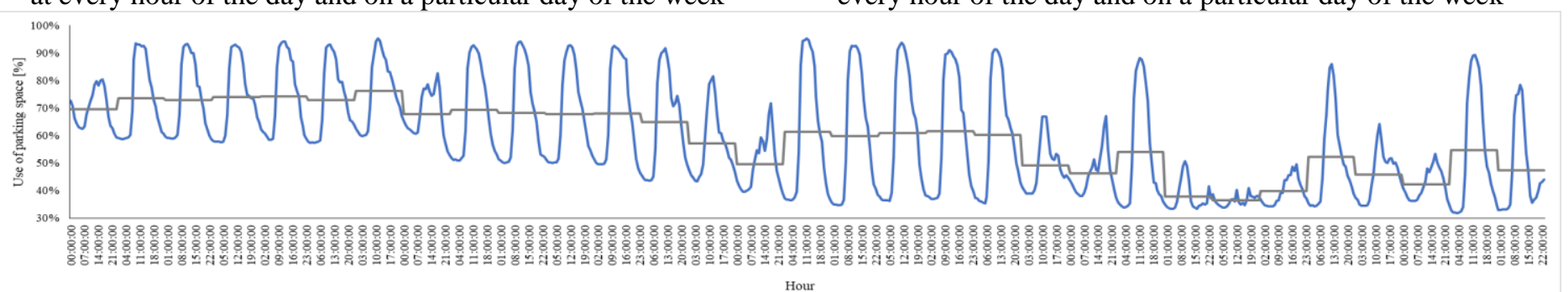

Fig. 6. Average use of parking space in all sections at every hour of the day and on every day in December

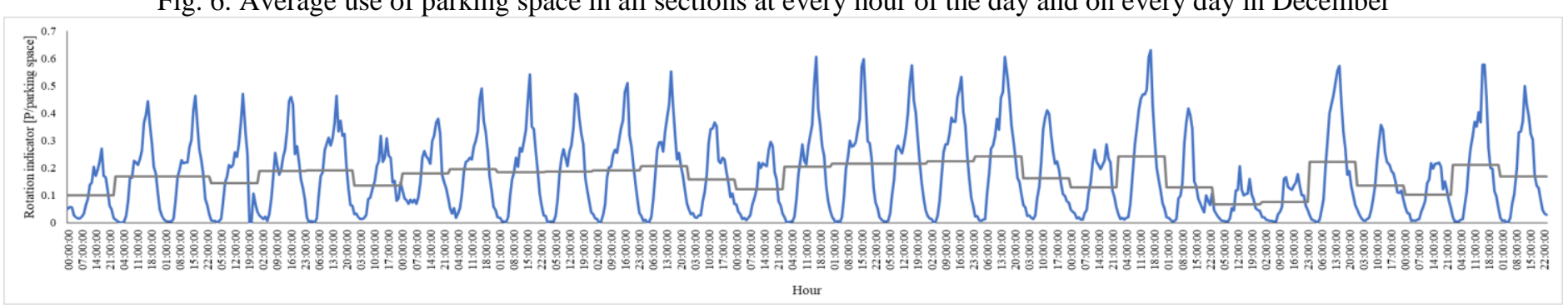

Fig. 7. Average rotation indicator in all sections at every hour of the day and on every day in December

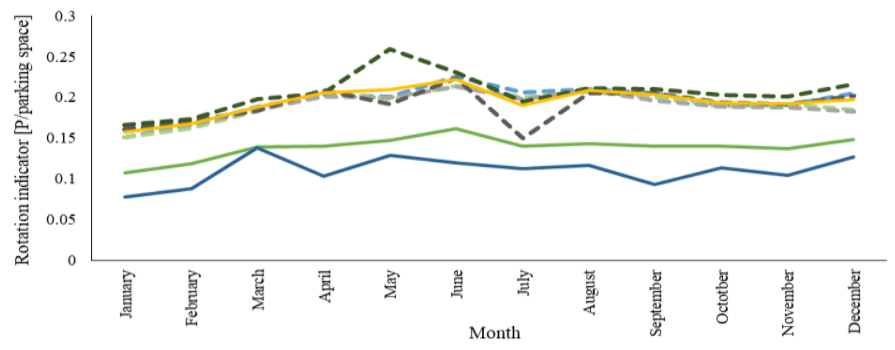

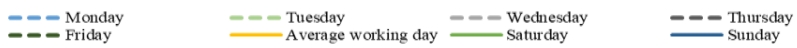

Fig. 3. Average rotation indicator in all sections in every month

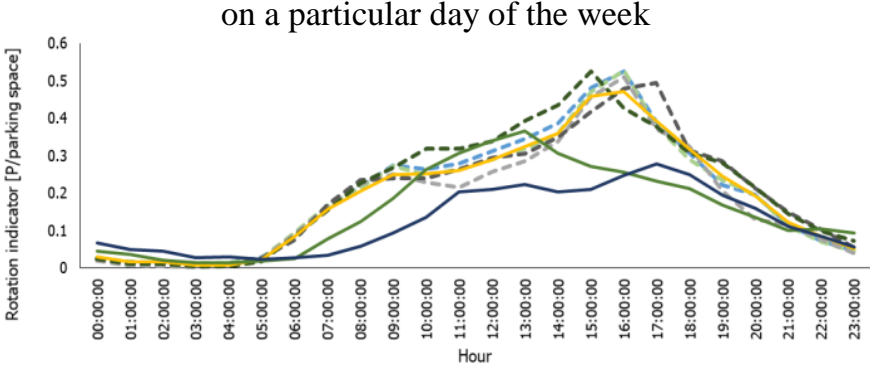

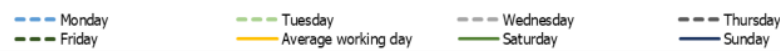

Fig. 5. Average rotation indicator in all sections in December at every hour of the day and on a particular day of the week 


\section{Conclusions}

The introduction of SPP and limited vehicle traffic in the centers of medium and large cities has become a necessity. This is caused i.a. due to the changing expectations of some residents, who perceive the city not only as a place where they take up work and take care of administrative formalities, but also a place for recreation, culture, and entertainment. Correct shaping of the transport policy, including the parking policy, is based on the information collected in measurements carried out in the field. Currently, apart from traditional measurement methods, the latest technologies are available, which enrich the available information sets with data collected in real-time. Knowing the available reserves and the largest number of parking spaces can contribute to the proper management of the parking space in the analysis area. The conducted analyzes allowed for the formulation of the following main conclusions:

- the greatest use of the parking space and the rotation indicator of the analyzed segments took place in December taking into account all days of the week,

- the greatest use of analyzed parking spaces occurred on working days, from 07:00 to 16:00 in December,

- the highest rotation indicator in analyzed parking spaces occurred on working days, from 09:00 to 18:00,

- the distribution of the rotation indicator during the day and week indicates that the drivers who use the analyzed parking are people whose purpose is e.g. official matters, shopping, family social meetings or dealing with short term issues in the city center,

- on 16-20.12.2020 (Monday-Friday), the use of parking space during the day was characterized by greater diversification than on the other days of the month. This fact may be related to the occurrence of Christmas the following week.

\section{Acknowledgments}

The present research has been financed from the "Excellence Initiative - Research University" program implemented at the Silesian University of Technology, 2020-2022 as a part of a grant entitled " Analysis of parking characteristics in the conditions of SPP and DIP functioning in selected areas of GZM cities".

\section{References}

[1] D. Shoup, "The high cost of free parking", J. of Planning Education and Research, vol. 17, no. 3, pp. 3-20, 1997.

[2] D. Shoup, "Cruising for parking", Transport Policy, vol. 13, Issue 6, pp. 479-486, 2006.

[3] U. Duda-Wiertel and A. Szarata, "The analysis of transport-related behaviours of drivers in highly occupied paid parking zones", Advances in Transportation Studies, vol. 47, pp. 35-48, 2019.

[4] E. Macioszek, "Changes in Values of Traffic Volume-Case Study Based on General Traffic Measurements in Opolskie Voivodeship (Poland)", in Directions of Development of Transport Networks and Traffic Engineering. Lecture Notes in Networks and Systems, Springer International Publishing Switzerland, vol. 51, pp. 66-76, 2019.

[5] A. Ibeas, R. Cordera, L. dell'Olio and J.L. Mora, "Modelling demand in restricted parking zones", Transportation Research Part A: Policy and Practice, vo. 45, issue 6, pp. 485-498.

[6] M. Borowska-Stefańska, S. Wiśniewski, "Designation of Paid Parking Zones in Łódz", Log. and Transp., vol.41, pp.57$71,2019$.

[7] E. Macioszek, "Electric Vehicles - Problems and Issues", in Smart and Green Solutions for Transport Systems. Advances in Intelligent Systems and Computing, Springer Nature Switzerland AG 2020, vol. 1091, pp. 169-183.

[8] E. Macioszek, P. Świerk and A. Kurek, "The Bike-Sharing System as an Element of Enhancing Sustainable Mobility A Case Study based on a City in Poland", Sustainability, vol. 12, pp. 1-29, 2020.

[9] H. Chai, R. Ma and H.M. Zhang, "Search for parking: A dynamic parking and route guidance system for efficient parking and traffic management", Journal of Intelligent Transportation Systems, vol. 23, issue 6, pp. 541-556, 2019.

[10] E. Macioszek, "E-mobility infrastructure in the Górnośląsko - Zagłębiowska Metropolis, Poland, and potential for development", in Proceedings of the $5^{\text {th }}$ World Congress on New Technologies (NewTech'19), Lisbon, 2019, vol. 108, pp. 1-4. 
[11] J. Simićević, S. Vukanovic and N. Milosavljević, "The effect of parking charges and time limit to car usage and parking behaviour", Transport Policy, vol. 30, pp. 125-131, 2013.

[12] E. Macioszek and A. Kurek, "The Use of a Park and Ride System-A Case Study Based on the City of Cracow (Poland)", Energies, vol. 13, pp. 1-26, 2020 